\title{
CRITICAL VALUES FOR A NONLINEAR DIFFERENCE EQUATION
}

\author{
A. BROWN ${ }^{1}$ \\ (Received 3 September 1985; revised 3 March 1986)
}

\begin{abstract}
The paper discusses equilibrium solutions and solutions with period two and period three for the difference equation

$$
x_{n+1}=Q+A x_{n} /\left(1+x_{n}^{2}\right),
$$

where $Q$ and $A$ are real, positive parameters. The equation was used by Bier and Bountis [1] as an example of a difference equation whose iteration diagram can show bubbles of finite length rather than the successive bifurcations usually expected. The paper examines in more detail what kind of solution can occur for given values of $Q$ and $A$ and establishes a series of critical curves which demarcate the regions in the $(Q, A)$ plane where solutions of period two or period three occur and the subregions where these periodic solutions are stable. This makes it easy to see how $Q$ and $A$ can be combined into a one-parameter equation which gives a bubble, or a series of bubbles, in the iteration diagram.
\end{abstract}

\section{Introduction}

A great deal of work has been done on first order difference equations which involve a single parameter [5, 8], mainly because of their relevance in physical and biological problems. One way of depicting the behaviour of the solutions is to produce an "iteration diagram", obtained by iterating the difference equation a large number of times, throwing away the first 50 or 100 results (to give the solution time to settle down) and plotting the next 100 against the parameter value that was used. This tends to pick out periodic solutions, if a stable periodic solution is available, although in practice only the solutions with shorter period are readily identifiable. The most familiar example is where, as the parameter

\footnotetext{
${ }^{1}$ Department of Theoretical Physics, Research School of Physical Sciences, Australian National University, G.P.O. Box 4, Canberra, A.C.T.2601.

(c) Copyright Australian Mathematical Society 1987, Serial-fee code 0334-2700/87
} 
increases, stable equilibrium solutions give way to stable solutions of period two and these in turn give way to stable solutions of period four, and so on, with the intervals of stability decreasing in size as these period-doubling bifurcations proceed. At a later stage solutions of odd period appear, culminating in solutions of period three, and after these solutions of period three have become unstable the iteration diagram shows a frothy and apparently chaotic structure. Periodic solutions can exist but with such brief intervals of stability that it is hard to pick them up on an iteration diagram.

More recent work has shown that other patterns can arise in the iteration diagram. In particular, the bifurcation process can be reversed at any stage. For example, we can have stable equilibrium solutions giving way to stable solutions of period two but with the latter replaced by stable equilibrium solutions again as the parameter increases, giving an elongated bubble in the iteration diagram. Diagrams of this kind are known from experimental work on RCL circuits with nonlinear capacitors $[4,7]$ and others have been obtained by starting from first order difference equations with two parameters, then introducing a relationship between the parameters which gives a one-parameter problem $[1,2,3,10]$. Oppo and Politi [10, Fig. 1] show clearly how this can be done and indeed it is evident from their diagram that it would be possible to have more than one reversal of the period-doubling process or, in terms of the iteration diagram, to have a string of bubbles as the parameter increases. Another possibility is to proceed through the usual sequence to the chaotic stage and then have a complete reversal as the parameter increases, with another interval of stable solutions of period three and eventually coalescence of solutions of period two to give equilibrium solutions. Examples of this arose in discussing the equation $[2,3]$

$$
y_{n+1}=2 k /\left\{1+\left(y_{n}-m\right)^{2}\right\}
$$

where $m$ and $k$ are real parameters, with $k>0$. This equation bears some resemblance to an example mentioned by Bier and Bountis [1]

$$
x_{n+1}=Q+\left\{A x_{n} /\left(1+x_{n}^{2}\right)\right\}
$$

where $Q$ and $A$ are real, positive parameters. In Figure 1 of their paper they show iteration diagrams for this equation where the period-doubling process has been reversed, in one case after solutions of period 8 had been attained. However their treatment is fairly brief and it is clear that more could be done to mark out the boundaries between different types of solution in the $(Q, A)$ plane. The present paper is intended to fill in some of these details.

Section 2 discusses the equilibrium solutions of equation (1.2) and their stability. It can be shown that there is exactly one positive solution, for any pair 
( $Q, A$ ), and that this solution is always stable. If we regard $Q$ as fixed and let $A$ increase, then at first the positive solution is the only equilibrium solution but for larger values of $A$ there are also two negative solutions. The larger of these (algebraically) is always unstable and the smaller solution is stable for $A_{0}(Q)<A$ $<A_{1}(Q)$, where $A_{0}(Q)$ and $A_{1}(Q)$ are tabulated for a number of values of $Q$. The curve $A=A_{0}(Q)$ in the $(Q, A)$ plane is the boundary between the region where there is only one real equilibrium solution and the region where there are three solutions.

In Section 3 it is shown that there are solutions with period two for $A>A_{1}(Q)$. These solutions are stable for $A>A_{1}(Q)$ when $0<Q<\sqrt{2}$ but for $Q>\sqrt{2}$ there is a finite interval of stability $A_{1}(Q)<A<A_{2}(Q)$. An expression for $A_{2}(Q)$ is obtained and some values of this function are tabulated.

Sections 4 and 5 deal with the more difficult problem of establishing similar boundary curves for solutions of period three. The lower boundary is a curve $A=A_{3}(Q)$, defined for $Q>2$, and for $A>A_{3}(Q)$ there are two solutions of period three. One solution is always unstable - and we can refer to these solutions as the unstable family-while the other solutions are stable over an interval $A_{3}(Q)<A<A_{4}(Q)$. These functions are tabulated for a number of values of $Q$. For an admissible pair $(Q, A)$, that is for $Q>2$ and $A>A_{3}(Q)$, the equations in Section 4 allow the elements of the period three solutions to be calculated, whether the solution is stable or unstable. This makes it feasible to follow the changes in the solutions in a much more complete way than would be possible from an iteration diagram.

Section 6 describes an attempt that was made to transform equation (1.2) into equation (1.1) by means of a bilinear relationship between $x_{n}$ and $y_{n}$. Suitable relationships were found but with restrictions that made them applicable only in certain regions of the $(Q, A)$ plane. This provided some extra information but not as much as had been hoped, since it did not allow the information about solutions of period three and period four for equation (1.1) to be taken over directly in discussing equation (1.2).

Although most of the calculations have been made on the basis that $Q$ is kept fixed and $A$ is allowed to vary, this was purely a matter of convenience. Generally speaking, the powers of $Q$ that come into various equations are greater than the powers of $A$, so it is better to fix $Q$ and have an equation of lower degree in $A$. Equation (5.1), which has powers of $Q$ up to $Q^{12}$ and powers of $A$ up to $A^{4}$, is an example of this. However, once the boundary curves have been established, different relationships between $A$ and $Q$ can be used to make equation (1.2) a one-parameter problem and this permits the solutions to change in a variety of different ways as the parameter increases. Some examples of this are mentioned in Section 7. 


\section{Equilibrium solutions}

If we write the Bier and Bountis equation as

$$
x_{n+1}=F\left(x_{n}\right)=Q+\left\{A x_{n} /\left(1+x_{n}^{2}\right)\right\},
$$

with $Q>0$ and $A>0$, then for any real $x_{n}$

$$
Q-(A / 2) \leqslant x_{n+1} \leqslant Q+(A / 2)
$$

and any periodic solution must have its elements in the interval $[Q-(A / 2), Q+$ $(A / 2)]$. So in looking for periodic solutions we can take $x_{0}$ in this interval and regard $F$ as a mapping of the interval into itself. In general, $F$ has two stationary points, at $x_{n}=-1$ and $x_{n}=+1$, but if we restrict $x_{0}$ to the interval above, then one or both of the stationary points can be excluded. More precisely, for $0<A<|2-2 Q|$ both stationary points are excluded, for $A \geqslant 2 Q+2$ both stationary points are included, and for other admissible values of $Q$ and $A$ there is one stationary point (at $x_{n}=1$ ). With this change in the number of stationary points we might expect some changes in the behaviour of the solutions in the different cases.

If $x_{n}=X$ is an equilibrium solution, then $X=F(X)$ and this gives a cubic equation

$$
G(X)=(X-Q)\left(1+X^{2}\right)-A X=0 .
$$

This equation has three real distinct roots [9] if and only if $A+(1 / 3) Q^{2}>1$ and $T(Q, A)>0$, where

$$
T(Q, A)=4 A^{3}+\left(Q^{2}-12\right) A^{2}+\left(12-20 Q^{2}\right) A-4\left(1+Q^{2}\right)^{2} .
$$

It can be shown that for $Q>0$ the equation $T(Q, A)=0$ has a unique positive solution for $A$, say $A_{0}(Q)$, with $A_{0}(Q)>1$. For $A>A_{0}(Q)$, the conditions $A+(1 / 3) Q^{2}>1$ and $T(Q, A)>0$ are both satisfied and hence there are three real distinct equilibrium solutions of equation (2.1) for $A>A_{0}(Q)$. For $0<A<$ $A_{0}(Q)$, there is a single equilibrium solution and the graph $A=A_{0}(Q)$, which is shown in Figure 1, serves as a critical curve separating the two regions in the $(Q, A)$ plane. As $Q \rightarrow 0$ from above, $A_{0}(Q) \rightarrow 1$, and for $Q$ large

$$
A_{0}(Q)=2 Q+2-\frac{1}{Q}+\frac{2}{Q^{2}}-\frac{21}{4 Q^{3}}+\frac{16}{Q^{4}}+O\left(Q^{-5}\right) .
$$

Some numerical values for $A_{0}(Q)$ are given in Table 1 .

The condition for local stability for an equilibrium solution, $X$, is that $\left|S_{1}\right|<1$ where

$$
S_{1}=F^{\prime}(X)=A\left(1-X^{2}\right) /\left(1+X^{2}\right)^{2} .
$$


TABLE 1. Values of $A_{0}(Q)$

[Equation (2.2) has 3 real solutions for $A>A_{0}(Q)$ ]

\begin{tabular}{c|cccccccc}
$Q$ & 0 & 0.5 & 1 & 2 & 3 & 5 & 10 & 20 \\
$A_{0}(Q)$ & 1.0 & 2.4864 & 3.6107 & 5.7290 & 7.7897 & 11.8535 & 21.9160 & 41.9544
\end{tabular}

It can be shown that equation (2.2) has exactly one positive root, say $X_{3}$, for any admissible pair $(Q, A)$ and that this positive root is always stable. For $A>A_{0}(Q)$ there are also two negative roots, say $X_{1}$ and $X_{2}$, with $X_{1}<X_{2}<0$. For $X=X_{2}, S_{1}>1$ and this equilibrium solution is always unstable. For $X=X_{1}$, the equilibrium solution is stable for $A_{0}(Q)<A<A_{1}(Q)$, where

$$
A_{1}(Q)=\frac{2}{Q^{2}}\left(1+Q^{2}\right)\left\{1+\sqrt{ }\left(1+Q^{2}\right)\right\}
$$

As $Q \rightarrow 0$ from above, $A_{1}(Q)$ becomes unbounded and for $Q$ large

$$
A_{1}(Q)=2 Q+2+\frac{3}{Q}+\frac{2}{Q^{2}}+\frac{3}{4 Q^{3}}+O\left(Q^{-5}\right)
$$

Some numerical values for $A_{1}(Q)$ are given in Table 2 and it appears as one of the critical curves in Figures 1 and 2. From equation (2.6), $A_{1}(Q)$ has a minimum value of 8 at $Q=\sqrt{3}$.

For $A=A_{0}(Q)$, the two negative equilibrium values coalesce to give a double root of equation (2.2) and $S_{1}=1$ for this double root. As $A$ increases from $A=A_{0}(Q)$ the value of $S_{1}$ corresponding to $X=X_{1}$ decreases. It becomes zero for $A=2 Q+2$ and -1 for $A=A_{1}(Q)$. Indeed the expression for $A_{1}(Q)$ was obtained from the condition that $S_{1}=-1$ when the equilibrium solution is on the point of becoming unstable. It can be shown that this occurs when

$$
X_{1}=-(1 / Q)\left\{1+\sqrt{ }\left(1+Q^{2}\right)\right\}
$$

and the corresponding value of $A$ follows from equation (2.2). If we take $A>A_{1}(Q)$ and follow $X_{1}$ as $A$ increases, with $Q$ fixed, then $X_{1}$ tends to $-\infty$ as $A \rightarrow \infty$ while the corresponding value of $S_{1}$ decreases to a minimum and then increases again, with $S_{1} \rightarrow-1$ as $A \rightarrow \infty$. Thus the equilibrium solution $X_{1}$ is unstable for $A>A_{1}(Q)$.

For the intermediate solution $X_{2}$, the value of $S_{1}$ increases as $A$ increases from $A=A_{0}(Q)$. For a given value of $Q,\left|X_{2}\right| \rightarrow 0$ and $S_{1} \rightarrow \infty$ as $A \rightarrow \infty$. However, it should be noted from equation (2.2) that $X=0$ cannot be an equilibrium solution for $Q>0$ and $A$ finite.

It can be shown that where there are three equilibrium solutions the product of the corresponding values of $S_{1}$ is

$$
P=(A-2-2 Q)(A-2+2 Q) / A
$$

and the sum of the $S_{1}$ values is $P+2$. These results were very useful in checking some of the numerical work. They also agree with the information available about superstable equilibrium solutions. From equation (2.5), $S_{1}=0$ for $X= \pm 1$ and it 
TABLE 2. Values of $A_{1}(Q)$ and $A_{2}(Q)$

[C2 solutions exist for $A>A_{1}(Q)$ and are stable for $A_{1}(Q)<A<A_{2}(Q)$ ]

\begin{tabular}{c|cccccccc}
$Q$ & 0.1 & 0.2 & 0.4 & 0.6 & 0.8 & 1.0 & 1.25 & 1.5 \\
$A_{1}(Q)$ & 405.01 & 105.03 & 30.117 & 16.367 & 11.688 & 9.657 & 8.531 & 8.097 \\
$A_{2}(Q)$ & - & - & - & - & - & - & - & 55.069 \\
$Q$ & 1.6 & 1.8 & 2.0 & 3.0 & 4.0 & 5.0 & 10.0 & 20.0 \\
$A_{1}(Q)$ & 8.029 & 8.007 & 8.090 & 9.250 & 10.887 & 12.686 & 22.321 & 42.155 \\
$A_{2}(Q)$ & 28.594 & 17.074 & 13.660 & 10.938 & 11.834 & 13.337 & 22.570 & 42.266
\end{tabular}

is easy to check that $X=-1$ implies $A=2 Q+2$ while $X=+1$ implies $A=2$ $-2 Q$. Along the line $A=2 Q+2$ in the $(Q, A)$ plane the smallest equilibrium solution is $X_{1}=-1$ and the other solutions can be written down explicitly. It can be checked that $P=0$ and the sum of the $S_{1}$ values is 2 in this case.

For $A=2-2 Q$ we have to add the condition $0<Q<1$ to ensure that $A>0$. This segment of the line $A=2-2 Q$ is shown in Figure 1 and it will be seen that it crosses the curve $A=A_{0}(Q)$ between $Q=0$ and $Q=0.5$, say for $Q=Q_{0}$. There will be three equilibrium solutions when $A=2-2 Q>A_{0}(Q)$, that is for $0<Q<Q_{0}$, but only one equilibrium solution, $X_{3}=1$, for $Q_{0}<Q$ $<1$. For $0<Q<Q_{0}$ we still have $X_{3}=1$ and the two negative solutions can be written down as explicit functions of $Q$. It can again be checked that $P=0$ and that the sum of the $S_{1}$ values is 2 . For $Q=Q_{0}$, the two negative solutions must be equal and this gives $Q_{0}=3-2 \sqrt{2}=0.1716$.

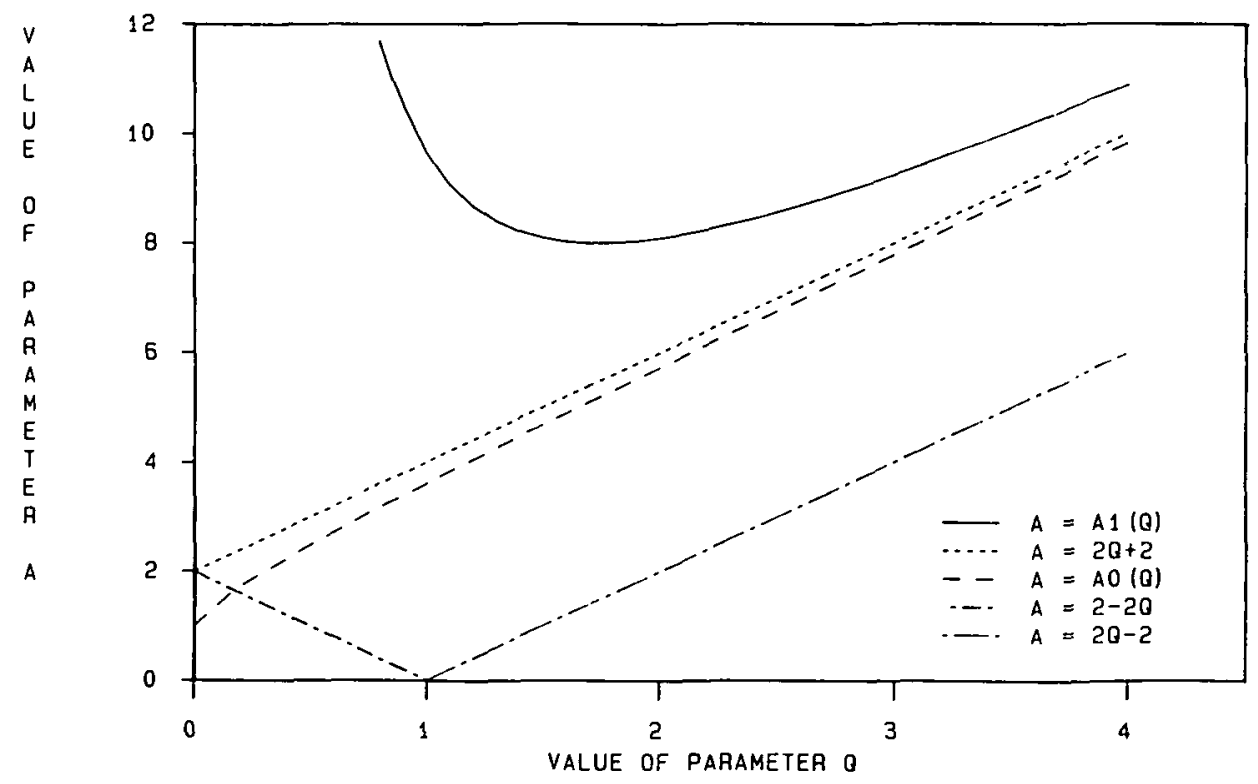

Figure 1. Graphs of $A 0(Q)$ and $A 1(Q)$ for $0<Q<4$. 


\section{Solutions with period two}

For a solution with minimum period two (referred to later as a $C 2$ solution) we want elements $b_{1}$ and $b_{2}$, with $b_{1} \neq b_{2}$ and with

$$
b_{2}=F\left(b_{1}\right), b_{1}=F\left(b_{2}\right) \text {. }
$$

These equations can be written as

$$
\begin{gathered}
\left(b_{2}-Q\right)\left(1+b_{1}^{2}\right)=A b_{1} \\
\left(b_{1}-Q\right)\left(1+b_{2}^{2}\right)=A b_{2},
\end{gathered}
$$

and their difference gives

$$
\left(b_{2}-b_{1}\right)\left\{1-b_{1} b_{2}+Q\left(b_{1}+b_{2}\right)\right\}=A\left(b_{1}-b_{2}\right) .
$$

If we write $\alpha=b_{1}+b_{2}$ and $\beta=b_{1} b_{2}$ and cancel the non-zero factor $b_{2}-b_{1}$, we get

$$
\beta=1+A+Q \alpha .
$$

Adding equations (3.2) and (3.3) and using the identity $b_{1}^{2}+b_{2}^{2}=\alpha^{2}-2 \beta$ gives

$$
\alpha(1-A)+\alpha \beta=2 Q+Q\left(\alpha^{2}-2 \beta\right) .
$$

We can use equation (3.5) to replace $\beta$ in equation (3.6) and thus obtain $\alpha$ in terms of $A$ and $Q$. The solution for $\beta$ follows and we get

$$
\alpha=-\frac{Q A}{1+Q^{2}}, \beta=\frac{1+A+Q^{2}}{1+Q^{2}} .
$$

The $C 2$ elements are then the roots of the equation

$$
b^{2}-\alpha b+\beta=0
$$

and the stability condition is that $\left|S_{2}\right|<1$, where

$$
S_{2}=F^{\prime}\left(b_{1}\right) F^{\prime}\left(b_{2}\right)=\frac{A^{2}\left(1-b_{1}^{2}\right)\left(1-b_{2}^{2}\right)}{\left[\left(1+b_{1}^{2}\right)\left(1+b_{2}^{2}\right)\right]^{2}} \text {. }
$$

To ensure that equation (3.8) has real distinct roots we must have $\alpha^{2}>4 \beta$ and this leads to the condition $A>A_{1}(Q)$, which we had before as the condition for the equilibrium solution $X_{1}$ to become unstable. From equation (3.7), $\alpha<0$ and $\beta>0$, so $b_{1}$ and $b_{2}$ must both be negative, and this supports the idea that the $C 2$ solution is a bifurcation of the unstable solution $X_{1}$.

We can obtain an alternative form for $S_{2}$ if we write

$$
\left(1-b_{1}^{2}\right)\left(1-b_{2}^{2}\right)=1-\left(\alpha^{2}-2 \beta\right)+\beta^{2}=(1+\beta)^{2}-\alpha^{2},
$$


then put $\alpha$ and $\beta$ in terms of $A$ and $Q$ from equation (3.7). The denominator in equation (3.9) can be dealt with in the same way and this leads to

$$
\begin{aligned}
S_{2} & =\frac{A^{2}\left\{(1+\beta)^{2}-\alpha^{2}\right\}}{\left\{(1-\beta)^{2}+\alpha^{2}\right\}^{2}}=\frac{\left(A+2+2 Q^{2}\right)^{2}-Q^{2} A^{2}}{A^{2}} \\
& =\left(1-Q^{2}\right)+4\left(1+Q^{2}\right) / A+4\left(1+Q^{2}\right)^{2} / A^{2} .
\end{aligned}
$$

For a fixed value of $Q, S_{2}$ will decrease as $A$ increases and as $A \rightarrow \infty$ the terms in $1 / A$ and $1 / A^{2}$ will tend to zero through positive values. Thus $S_{2} \rightarrow 1-Q^{2}$ from above. For $A=A_{1}(Q), S_{2}=1$ and the solutions will be stable as $A$ increases until $S_{2}$ becomes equal to -1 . This is only possible if $Q>\sqrt{2}$. For $0<Q<\sqrt{2}$ the $C 2$ solutions are stable for all $A>A_{1}(Q)$. For $Q>\sqrt{2}$ there will be a critical value of $A$, say $A_{2}(Q)$, at which $S_{2}=-1$, with stable solutions for $A_{1}(Q)<A<A_{2}(Q)$ and unstable solutions for $A>A_{2}(Q)$. From equation (3.11), $S_{2}=-1$ when

$$
\left(A+2+2 Q^{2}\right)^{2}-Q^{2} A^{2}=-A^{2}
$$

and this gives

$$
A_{2}(Q)=\frac{2\left(1+Q^{2}\right)\left\{1+\sqrt{ }\left(Q^{2}-1\right)\right\}}{Q^{2}-2} .
$$

Table 2 includes some values of $A_{2}(Q)$ and Figure 2 includes graphs of $A=A_{1}(Q)$ and $A=A_{2}(Q)$. As $Q \rightarrow \sqrt{2}$ from above, $A_{2}(Q) \rightarrow \infty$ and the table indicates a minimum around $Q=3$. More precisely, the minimum is $A_{2}=10.9282$ for $Q=2.9093$. For $Q$ large,

$$
A_{2}(Q)=2 Q+2+\frac{5}{Q}+\frac{6}{Q^{2}}+\frac{35}{4 Q^{3}}+\frac{12}{Q^{4}}+o\left(Q^{-5}\right)
$$

and hence

$$
\begin{aligned}
A_{2}(Q)-A_{1}(Q) & =\text { width of stability interval } \\
& =\frac{2}{Q}+\frac{4}{Q^{2}}+\frac{8}{Q^{3}}+\frac{12}{Q^{4}}+O\left(Q^{-5}\right) .
\end{aligned}
$$

It was noted in Section 2 that the positive equilibrium solution $X_{3}$ exists and is stable for all positive values of $A$ and $Q$. This means that it is possible to have a stable $C 2$ solution and a stable equilibrium value for the same pair $(Q, A)$, with an iteration diagram which is sensitive to the choice of $x_{0}$. For example, $x_{0} \geqslant 0$ makes $x_{n}>0$ for $n=1,2,3, \ldots$ and in this case it would be impossible to pick up the $C 2$ solution. Indeed this could happen with $x_{0}<0$ also. For example, if $Q=5$ and $A=13$ there is a superstable $C 2$ solution with $b_{1}=-1.0$ and $b_{2}=-1.5$. If we take $x_{0}=-0.4$, we get $x_{1}=+0.5172$ and then $x_{n}>0$ for $n=2,3,4, \ldots$. After a few iterations $x_{n}$ is close to the stable equilibrium solution $X_{3}=6.8565$. 


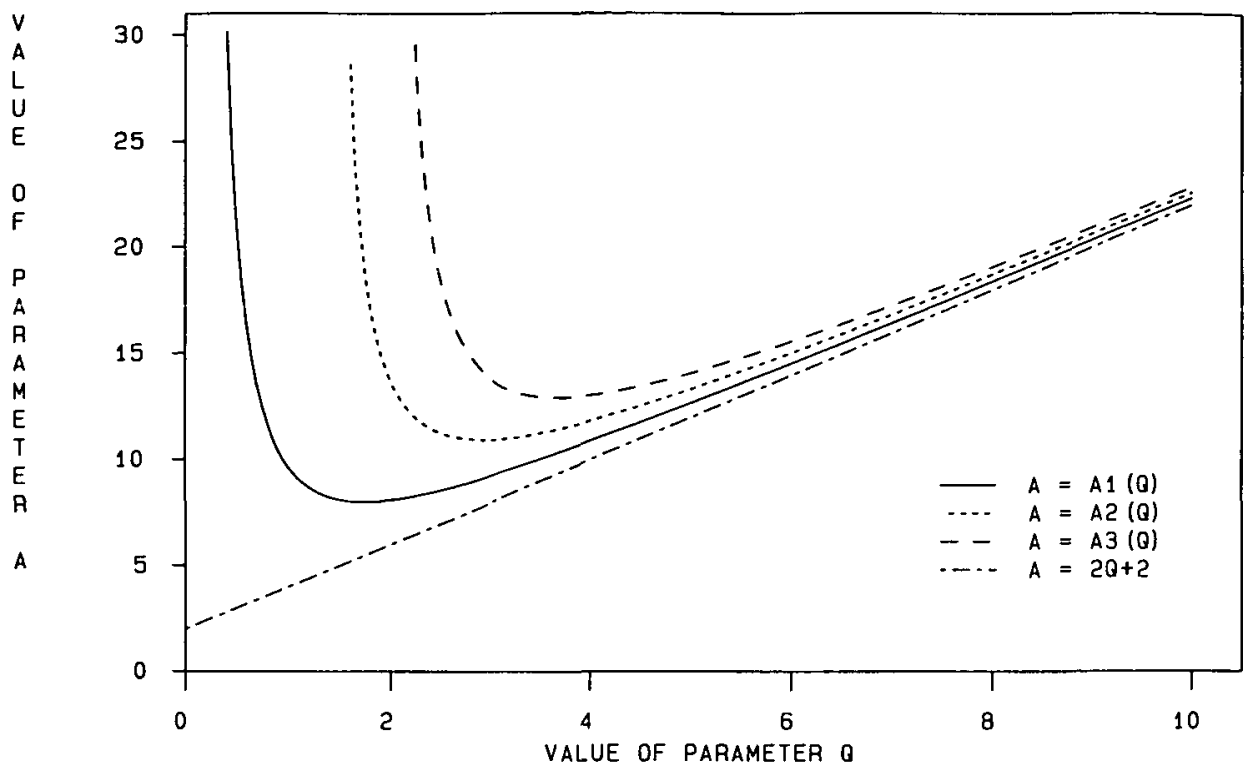

Figure 2. Graphs of $A 1(Q), A 2(Q)$ and $A 3(Q)$.

In the same way, the work in Sections 4 and 5 shows that it is possible to have a stable solution of period three for suitable parameter values $Q$ and $A$. There must also be a stable equilibrium solution, $X_{3}$, and the iteration diagram would be sensitive to the choice of $x_{0}$.

\section{Solutions with period three: basic equations}

In Section 3 the determination of $C 2$ solutions was made easier by using symmetrical functions of the solution elements and relating these to the parameters $Q$ and $A$. For solutions with minimum period three ( $C 3$ solutions) the same approach can be used. The analysis follows the same lines as for equation (1.1) [2, Section 4] so most of the detailed derivations are omitted in the account below.

For a $C 3$ solution we want real distinct elements $b_{1}, b_{2}, b_{3}$ which satisfy the equations

$$
b_{2}=F\left(b_{1}\right), b_{3}=F\left(b_{2}\right), b_{1}=F\left(b_{3}\right) \text {. }
$$

Rather than form an equation for, say, $b_{1}$ directly it is convenient to make use of the symmetrical functions

$$
\alpha=b_{1}+b_{2}+b_{3}, \beta=b_{1} b_{2}+b_{2} b_{3}+b_{3} b_{1}, \gamma=b_{1} b_{2} b_{3},
$$


and to construct equations which give $\alpha, \beta, \gamma$ for a given pair of parameter values $(Q, A)$. The solution elements are then the roots of the cubic

$$
h(b)=b^{3}-\alpha b^{2}+\beta b-\gamma=0 \text {. }
$$

To illustrate the procedure we note that equation (4.1) gives

$$
b_{1}^{2} b_{2}=-b_{2}+Q+Q b_{1}^{2}+A b_{1}
$$

and two similar equations obtained by cyclic permutation of the suffixes. If we add these equations

$$
\sum b_{1}^{2} b_{2}=-\alpha+3 Q+Q\left(\alpha^{2}-2 \beta\right)+A \alpha,
$$

where $\sum$ is used to denote cyclic summation over the suffixes 1,2 , and 3 . If we multiply equation (4.4) by $b_{3}$ and sum cyclically we get

$$
\alpha \gamma=-\beta+Q \alpha+Q \Sigma b_{1} b_{2}^{2}+A \beta
$$

and we can use the identity

$$
\sum b_{1}^{2} b_{2}+\sum b_{1} b_{2}^{2}=\alpha \beta-3 \gamma
$$

to eliminate $\sum b_{1}^{2} b_{2}$ and $\sum b_{1} b_{2}^{2}$. The result is an equation of the form

$$
a_{11} \beta+a_{12} \gamma=a_{13} \text {, }
$$

with

$$
\begin{aligned}
& a_{11}=(A-1)+\alpha Q+2 Q^{2}, \\
& a_{12}=-\alpha-3 Q, \\
& a_{13}=\left(\alpha^{2}+3\right) Q^{2}+(A-2) Q \alpha .
\end{aligned}
$$

Similar algebraic manipulation gives a second linear equation

$$
a_{21} \beta+a_{22} \gamma=a_{23} \text {, }
$$

with

$$
\begin{aligned}
& a_{21}=(1-A)+(3+A) Q^{2}+2 Q^{4}, \\
& a_{22}=3 A Q+\alpha\left(1+Q^{2}\right), \\
& a_{23}=\left(\alpha^{2}+3\right) Q^{2}\left(1+A+Q^{2}\right)+A Q \alpha\left(A+Q^{2}\right) .
\end{aligned}
$$

From these two linear equations

$$
\beta D(\alpha, Q, A)=D_{1}(\alpha, Q, A), \quad \gamma D(\alpha, Q, A)=D_{2}(\alpha, Q, A),
$$

where

$$
\begin{gathered}
D(\alpha, Q, A)=\left(1+Q^{2}\right) \alpha^{2}+\left(5 A+4+4 Q^{2}\right) Q \alpha \\
+3\left\{(A-1)^{2}+(3+3 A) Q^{2}+2 Q^{4}\right\} \\
D_{1}(\alpha, Q, A)=\left(A+2+2 Q^{2}\right) Q \alpha^{3}+\left\{\left(A^{2}+A-2\right)+(1+8 A) Q^{2}+3 Q^{4}\right\} \alpha^{2} \\
+3\left\{\left(2 A^{2}-A+2\right)+(A+2) Q^{2}\right\} Q \alpha+9 Q^{2}\left(2 A+1+Q^{2}\right),
\end{gathered}
$$




$$
\begin{aligned}
D_{2}(\alpha, Q, A)= & \left(A+1+Q^{2}\right) Q^{2} \alpha^{3}+\left\{\left(2 A^{2}+A-2\right)+(3 A-2) Q^{2}\right\} Q \alpha^{2} \\
& +\left\{\left(A^{3}-3 A+2\right)+\left(2 A^{2}+A+9\right) Q^{2}+7 Q^{4}\right\} \alpha \\
& +3 Q\left\{\left(A^{2}+A-2\right)+(2 A-2) Q^{2}\right\} .
\end{aligned}
$$

In general, these equations give $\beta$ and $\gamma$, once $\alpha, Q, A$ are known. The main problem that remains is to find an equation for $\alpha$, for given values of $Q$ and $A$.

It can be shown (along the lines indicated above) that

$$
\begin{aligned}
\beta^{2}=(A & \left.+2-2 Q^{2}\right) \beta+2 \alpha \gamma+\left(Q^{2}-1\right) \alpha^{2}+Q A \alpha+3 Q^{2}, \\
\beta \gamma= & 3 Q \beta+(3 A+Q \alpha) \gamma+(1-A) \alpha-\left(\alpha^{2}+3\right) Q, \\
3 \gamma^{2}= & \left(5 Q^{2}-2 A-1\right) \beta+\left\{3 A Q+\left(A+1+Q^{2}\right) \alpha\right\} \gamma \\
& +\left(1-2 Q^{2}\right) \alpha^{2}-2 Q A \alpha-6 Q^{2},
\end{aligned}
$$

so one of these equations could be used with equation (4.12) to eliminate $\beta$ and $\gamma$, thus leaving a relationship between $\alpha, Q$ and $A$. However this would involve expansions for $D^{2}, D D_{1}, D D_{2}$ and similar products. Instead, a third linear equation for $\beta$ and $\gamma$ was obtained by multiplying equation (4.8) by $3 \gamma$ and substituting for $\beta \gamma$ and $3 \gamma^{2}$ from equations (4.17) and (4.18). This gives

$$
a_{31} \beta+a_{32} \gamma=a_{33},
$$

with

$$
\begin{aligned}
& a_{31}=\left(1+2 A+4 Q^{2}\right) \alpha+\left(15 A-6+3 Q^{2}\right) Q \\
& a_{32}=9(A-1)\left(A+Q^{2}\right)+3 Q\left(A+Q^{2}\right) \alpha-\left(A+1+Q^{2}\right) \alpha^{2}, \\
& a_{33}=9 Q(A-1)+3 \alpha\left\{(A-1)^{2}-Q^{2}\right\}+(4 A-3) Q \alpha^{2}+\left(1+Q^{2}\right) \alpha^{3} .
\end{aligned}
$$

From equations (4.8), (4.10) and (4.19), the equation for $\alpha$ is

$$
Q E(\alpha, Q, A)=\operatorname{det}\left|a_{i j}\right|=0,
$$

where $E(\alpha, Q, A)$ is a polynomial in $\alpha$ of degree 5 . At most there should be two $C 3$ solutions for a given pair $(Q, A)$, so the equation for $\alpha$ should be a quadratic. The discrepancy arises because the analysis did not use the condition $b_{1} \neq b_{2} \neq b_{3}$ to rule out the degenerate case $b_{1}=b_{2}=b_{3}=X$, where $X$ is an equilibrium solution. If we put $X=\alpha / 3$ in equation (2.2) we get

$$
0=\alpha^{3}-3 Q \alpha^{2}+9(1-A) \alpha-27 Q=E_{0}(\alpha, Q, A)
$$

and it can be verified that $E(\alpha, Q, A)$ includes $E_{0}(\alpha, Q, A)$ as a factor. The co-factor has the form

$$
E_{1}(\alpha, Q, A)=q_{0} \alpha^{2}+q_{1} \alpha+q_{2},
$$


with

$$
\begin{gathered}
q_{0}=\left(1+Q^{2}\right)^{3}+2 A Q^{2}\left(1+Q^{2}\right)+A^{2} Q^{2} \\
q_{1}=Q A\left\{3\left(1+Q^{2}\right)^{2}+A\left(1+5 Q^{2}\right)+2 A^{2}\right\} \\
q_{2}=(A-1)\left(A^{3}-1\right)+3 Q^{2}\left(1+A^{2}+A^{3}\right)+Q^{4}\left(3+A+2 A^{2}\right)+Q^{6}
\end{gathered}
$$

It will be seen that $q_{0}, q_{1}, q_{2}$ can easily be evaluated for any pair $(Q, A)$ and for the $C 3$ solutions $\alpha$ is determined by the quadratic equation $q_{0} \alpha^{2}+q_{1} \alpha+q_{2}=0$. For each value of $\alpha$, equation (4.12) then gives $\beta$ and $\gamma$ and the elements $b_{1}, b_{2}, b_{3}$ can be found from (4.3). Thus the equations involved are relatively simple and they apply whether the solutions are stable or unstable. Indeed the stability can be tested readily as part of the computational scheme. The condition for stability is that $\left|S_{3}\right|<1$, where

$$
S_{3}=F^{\prime}\left(b_{1}\right) F^{\prime}\left(b_{2}\right) F^{\prime}\left(b_{3}\right)=\frac{A^{3}\left(1-b_{1}^{2}\right)\left(1-b_{2}^{2}\right)\left(1-b_{3}^{2}\right)}{\left\{\left(1+b_{1}^{2}\right)\left(1+b_{2}^{2}\right)\left(1+b_{3}^{2}\right)\right\}^{2}} \text {. }
$$

Since $S_{3}$ is a symmetrical function of $b_{1}, b_{2}, b_{3}$ it can be expressed in terms of $\alpha, \beta, \gamma$ and with the help of equation (4.1) it can be shown that

$$
S_{3}=\frac{(1+\alpha+\beta+\gamma)(1-\alpha+\beta-\gamma)}{\left(1-\beta+\alpha \gamma-\gamma^{2}\right)} .
$$

This enables $S_{3}$ to be calculated without knowing $b_{1}, b_{2}, b_{3}$ explicitly.

For the most part the computations are straightforward, although some special cases arise and they are included in the discussion in the next section.

\section{Solutions with period three: discussion}

From equations (4.24), (4.25) and (4.26) $q_{0}, q_{1}$ and $q_{2}$ are positive for $Q>0$ and $A>0$, so we can deduce that $\alpha<0$ for any real solutions of $q_{0} \alpha^{2}+q_{1} \alpha+$ $q_{2}=0$. This rules out a $C 3$ solution with $b_{1} \geqslant 0$, for equation (2.1) would then give $b_{2}>0, b_{3}>0$, and hence $\alpha>0$. Our choice of which element we call $b_{1}$ is arbitrary so this argument shows that $b_{i}<0$ for each $C 3$ element. In particular, to obtain a superstable solution we can start with $b_{1}=-1$ (which gives $F^{\prime}\left(b_{1}\right)=0$ and $S_{3}=0$ ), work out $b_{2}$ and $b_{3}$ and impose the condition that $F\left(B_{3}\right)=-1$. For $Q=3$, for example, the cyclic condition gave $A=13.80712906$ and from this $b_{2}=-3.90356454, b_{3}=-0.31922834$. Apart from serving as a typical $C 3$ solution, this example was of great value in checking the algebra in Section 4. 
If we set $H_{1}(Q, A)=q_{1}^{2}-4 q_{0} q_{2}$, then equation (4.29) has two real roots for $H_{1}(Q, A)>0$ and complex roots for $H_{1}(Q, A)<0$, so we can expect $H_{1}(Q, A)=$ 0 to provide the boundary curve $A=A_{3}(Q)$ where $C 3$ solutions first appear. It is convenient here to use $R=1+Q^{2}$ and to write $H_{1}(Q, A)=H(R, A)$. With this minor change of notation

$$
\begin{aligned}
H(R, A)= & A^{4}(R-2)^{2}(R-5)+2 R A^{3}(R-2)\left(R^{2}-7 R+4\right) \\
& +A^{2} R^{2}(R-1)\left(R^{2}-16 R+16\right)+A R^{4}(16-12 R)-4 R^{6} \\
= & \left\{(R-2) A+R^{2}\right\}^{2}\left\{(R-5) A^{2}-4 R A-4 R^{2}\right\} .
\end{aligned}
$$

For a given value of $Q, H(R, A)=0$ when $A$ is given by either

$$
(R-2) A+R^{2}=0
$$

For $A>0,(R-2) A+R^{2}>0$ for $R \geqslant 2$ and this means that equation (5.3) can only be satisfied when $R<2$, that is when $0<Q<1$. Since all $C 2$ solutions are stable for $0<Q<1$ it seems unlikely that there will be $C 3$ solutions in this region and this can be checked if we assume that $0<Q<1$ and take

$$
A=R^{2} /(2-R)=\left(1+Q^{2}\right)^{2} /\left(1-Q^{2}\right) \text {. }
$$

This makes $q_{1}^{2}=4 q_{0} q_{2}$, so the quadratic for $\alpha$ has a double root $-q_{1} /\left(2 q_{0}\right)$. If we substitute for $A$ in $q_{0}$ and $q_{1}$, the result is

$$
Q \alpha=\frac{(1-R)(2+R)}{2-R}=1-A .
$$

We now have $\alpha$ and $A$ in terms of $Q$ and with these expressions equation (4.8) reduces to

$$
\left(1-Q^{2}\right) \beta+2 Q \gamma=3-Q^{2}
$$

Equations (4.10) and (4.19) give exactly the same equation so this is a case where $D(\alpha, Q, A)$ is zero and the usual method of solution for $\beta$ and $\gamma$ breaks down.

We can obtain an equation for $\beta$ from equation (4.16), by using equation (5.7) to replace $2 \alpha \gamma$ by terms involving $\beta$. This leads to a quadratic

$$
\left(1-Q^{2}\right)^{2} \beta^{2}-2 \beta\left(1-Q^{2}\right)\left(Q^{4}-2 Q^{2}+3\right)+9+9 Q^{4}-2 Q^{6}=0,
$$

and the condition for real roots is that $D_{3} \geqslant 0$, where

$$
D_{3}=Q^{2}\left(Q^{2}-3\right)\left(Q^{4}+Q^{2}+4\right) \text {. }
$$

Thus the equation for $\beta$ gives complex roots for $0<Q<1$ and we have checked that equation (5.3) does not lead to real $C 3$ solutions for $A>0$. 
The alternative condition for $H(R, A)=0$ is that equation (5.4) holds. We must have $R>5$, that is $Q>2$, to give a positive solution for $A$. The appropriate solution is then

$$
A=\frac{2 R\{1+\sqrt{ }(R-4)\}}{R-5}=\frac{2\left(1+Q^{2}\right)\left\{1+\sqrt{ }\left(Q^{2}-3\right)\right\}}{Q^{2}-4} .
$$

If we call this function $A_{3}(Q)$, then $A=A_{3}(Q)$ gives the boundary curve we are seeking, with real $C 3$ solutions for $A \geqslant A_{3}(Q)$. Some values of $A_{3}(Q)$ are given in Table 3. As $Q \rightarrow 2, A_{3} \rightarrow \infty$ and for $Q$ large

$$
A_{3}(Q)=2 Q+2+\frac{7}{Q}+\frac{10}{Q^{2}}+\frac{91}{4 Q^{3}}+\frac{40}{Q^{4}}+O\left(Q^{-5}\right) .
$$

$A_{3}$ has a minimum value 12.944272 at $Q=3.670441$. A graph of $A=A_{3}(Q)$ is included in Figure 2.

For $A=A_{3}(Q)$, the quadratic for $\alpha$ has a double root. It is possible to write down expressions for $q_{0}, q_{1}, q_{2}, \alpha$ and $D(\alpha, Q, A)$ in terms of $R$ but these expressions are too cumbrous to provide additional information. However the numerical evidence is that $D$ is non-zero and that there is a single real $C 3$ solution for $A=A_{3}(Q)$, with $S_{3}=1$ for this solution. For $A>A_{3}(Q)$ there are two $C 3$ solutions for each pair $(Q, A)$. For a given value of $Q, S_{3}$ increases as $A$ increases for the solution with the smaller value of $\alpha$ and all solutions of this family are unstable. For the other family, which we can refer to as the stable family of solutions, $S_{3}$ decreases as $A$ increases and there is a finite interval within which these solutions are stable. The upper limit of stability occurs when $S_{3}=-1$ and we use $A_{4}(Q)$ for the corresponding value of $A$. Values of $A_{4}(Q)$ were determined numerically and are included with $A_{3}(Q)$ in Table 3. It will be seen that the width of the stability interval decreases as $Q$ increases, from 0.139 at $Q=2.1$ to 0.014 at $Q=5.0$ and 0.0035 at $Q=20.0$. We can guess that $A_{4}(Q)$ also behaves like $2 Q+2$ as $Q \rightarrow \infty$, although we have no analytical evidence for this. Because the difference $A_{4}(Q)-A_{3}(Q)$ is so small, a separate graph for $A_{4}(Q)$ was not included in Figure 2. It would be difficult to distinguish $A_{4}(Q)$ from $A_{3}(Q)$ on the scale of this diagram.

TABLE 3. Values of $A_{3}(Q)$ and $A_{4}(Q)$

\begin{tabular}{|c|c|c|c|c|c|c|}
\hline$Q$ & 2.1 & 2.3 & 2.5 & 3.0 & 3.5 & 4.0 \\
\hline$A_{3}(Q)$ & 57.7269 & 24.5093 & 18.0623 & 13.7980 & 12.9814 & 13.0491 \\
\hline$A_{4}(Q)$ & 57.8662 & 24.5941 & 18.1226 & 13.8330 & 13.0064 & 13.0688 \\
\hline$Q$ & 5.0 & 7.0 & 8.0 & 10.0 & 20.0 & \\
\hline$A_{3}(Q)$ & 14.0906 & 17.2941 & 19.0889 & 22.8278 & 42.3781 & \\
\hline$A_{4}(Q)$ & 14.1048 & 17.3037 & 19.0972 & 22.8345 & 42.3816 & \\
\hline
\end{tabular}

[C3 solutions exist for $A>A_{3}(Q)$, with stable solutions for $A_{3}(Q)<A<A_{4}(Q)$ ] 
As $A$ approaches $A_{3}(Q)$ from above, the stable solutions and the unstable solutions approach a common limit, the solution for $A=A_{3}(Q)$. For $A$ slightly greater than $A_{3}(Q)$ the elements of the two solutions will not differ greatly and indeed this is true even at $A=A_{4}(Q)$, as indicated by the examples of $C 3$ solutions in Table 4. In each case the smallest solution element has been taken as $b_{1}$ and the value of $S_{3}$ is given in the last column to show which is the stable solution and which is the unstable solution.

\begin{tabular}{|c|c|c|c|c|c|c|}
\hline$Q$ & A & $\alpha$ & & $b_{1}, b_{2}, b_{3}$ & & $S_{3}$ \\
\hline 2.5 & 18.122596 & $\begin{array}{l}-7.743 \\
-7.807\end{array}$ & $\begin{array}{l}-6.560, \\
-6.549\end{array}$ & $\begin{array}{l}-0.200 \\
-0.204\end{array}$ & $\begin{array}{l}-0.983 \\
-1.054\end{array}$ & $\begin{array}{r}-1.000002 \\
+2.856081\end{array}$ \\
\hline 5.0 & 14.104848 & $\begin{array}{l}-3.592 \\
-3.658\end{array}$ & $\begin{array}{l}-2.052 \text {, } \\
-2.045\end{array}$ & $\begin{array}{l}-0.555 \\
-0.566,\end{array}$ & $\begin{array}{l}-0.985 \\
-1.047\end{array}$ & $\begin{array}{r}-0.999964 \\
+2.814133\end{array}$ \\
\hline 10.0 & 22.834487 & $\begin{array}{l}-3.166 \\
-3.207\end{array}$ & $\begin{array}{l}-1.417 \\
-1.413\end{array}$ & $\begin{array}{l}-0.758, \\
-0.767\end{array}$ & $\begin{array}{l}-0.991 \\
-1.027\end{array}$ & $\begin{array}{r}-0.999999 \\
+2.821887\end{array}$ \\
\hline 20.0 & 42.381568 & $\begin{array}{l}-3.058 \\
-3.082\end{array}$ & $\begin{array}{l}-1.191 \\
-1.188,\end{array}$ & $\begin{array}{l}-0.873 \\
-0.879\end{array}$ & $\begin{array}{l}-0.995 \\
-1.015\end{array}$ & $\begin{array}{r}-1.000165 \\
+2.833216\end{array}$ \\
\hline
\end{tabular}

\section{Bilinear transformation of Bier and Bountis equation}

As mentioned in Section 1 there are some similarities between equations (1.1) and (1.2) and this raised the question whether it would be possible to go from one to the other by a suitable transformation, so that the results obtained for equation (1.1) could be used in the discussion of equation (1.2). With this in mind, a bilinear transformation of equation (1.2) was examined. If we write

$$
z_{n}=L\left(x_{n}\right)=\left(a+b x_{n}\right) /\left(c+x_{n}\right) \quad(a \neq b c)
$$

then the inverse relationship is

$$
x_{n}=\left(a-c z_{n}\right) /\left(z_{n}-b\right)
$$

and equation (1.2) gives

$$
z_{n+1} J_{2}\left(z_{n}\right)=J_{1}\left(z_{n}\right)
$$

where $J_{1}$ and $J_{2}$ are in general quadratics in $z_{n}$. In order to put this into the form of equation (1.1), $J_{1}$ must have no terms in $z_{n}$ and $z_{n}^{2}$ and this gives two conditions on $a, b, c$, namely

$$
\begin{gathered}
b c A=(a+b Q)\left(1+c^{2}\right), \\
b A(a+b c)=2(a+b Q)(a c+b) .
\end{gathered}
$$


It is easy to show that $a+b Q$ cannot be zero and if $a+b Q \neq 0$ then $b$ and $c$ are non-zero, from equation (6.4). (As usual, we shall assume that $A>0$ and $Q>0$.) It follows that

$$
\frac{a+b c}{c}=\frac{2(a c+b)}{1+c^{2}}
$$

and hence

$$
(b c-a)\left(1-c^{2}\right)=0 .
$$

Since $b c \neq a$, we get $c= \pm 1$ and we can consider the two cases separately.

When $c=1$, we have $2(a+b Q)=b A$, that is

$$
a=\frac{1}{2} b(A-2 Q), \quad b-a=\frac{1}{2} b(2+2 Q-A) .
$$

After some reduction, equation (6.3) becomes

$$
z_{n+1}\left[\left\{z_{n}-\frac{1}{2}(a+b)\right\}^{2}+M^{2}\right]=\frac{1}{4} b^{2} A(b-a),
$$

where

$$
M^{2}=\frac{1}{8} b(b-a)(A+2 Q+2) .
$$

To make $k>0$ we must have $b-a>0$ in equation (6.9) and for $M$ to be real we must then have $b>0$. Equation (6.8) now gives $2+2 Q-A>0$, that is $0<A<2 Q+2$, which is an important restriction on $A$. With this restriction we can write

$$
\begin{gathered}
M=\left(\frac{1}{4} b\right)_{V}\left\{(2+2 Q)^{2}-A^{2}\right\} \\
m=\frac{a+b}{2 M}=\frac{b(A+2-2 Q)}{4 M}, \\
k=\frac{b A}{M(2+2 Q+A)}, \\
z_{n}=M y_{n}
\end{gathered}
$$

and equation (6.9) goes over to the form

$$
y_{n+1}\left\{1+\left(y_{n}-m\right)^{2}\right\}=2 k,
$$

as required. Note that a precise value for $b$ is not needed. If we write

$$
M_{0}=4 M / b=\sqrt{ }\left\{(2+2 Q)^{2}-A^{2}\right\},
$$

then the transformation becomes

$$
y_{n}=\frac{4 x_{n}+2 A-4 Q}{\left(x_{n}+1\right) M_{0}}
$$


with

$$
M_{0} m=A+2-2 Q, \quad M_{0} k=4 A /(2+2 Q+A) .
$$

With this mapping, equilibrium points of equation (1.2) will map into equilibrium points of equation (1.1), and vice versa, and the stability testing function (STF) will be the same for corresponding equilibrium points. From equation (6.1), if $x_{n+1}=x_{n}$, then $z_{n+1}=z_{n}$ and $y_{n+1}=y_{n}$ from equation (6.14). If $x_{n}=X$ is an equilibrium point of equation (1.2) and $z_{n}=Z$ is the corresponding equilibrium point of equation (6.9), we obtain the STF at $Z$ by evaluating $\left(d z_{n+1}\right) /\left(d z_{n}\right)$ at $z_{n}=Z=L(X)$. We can write

$$
\frac{d z_{n+1}}{d z_{n}}=\frac{d z_{n+1}}{d x_{n+1}} \frac{d x_{n+1}}{d x_{n}} \frac{d x_{n}}{d z_{n}}=L^{\prime}\left(x_{n+1}\right) F^{\prime}\left(x_{n}\right) / L^{\prime}\left(x_{n}\right)
$$

and evaluating these derivatives at $x_{n}=x_{n+1}=X$ gives

$$
S_{1}^{*}=S T F \text { at } Z=F^{\prime}(X)=S_{1} .
$$

The change of scale from $z_{n}$ to $y_{n}$ does not alter the STF; indeed the same argument would apply. The only problem that could arise would be if $x_{n}=X$ is a singular point of the transformation. For $c=1$, there is a singular point at $x_{n}=-1$ but we know that $X=-1$ implies $A=2 Q+2$ and this is excluded by the restriction $0<A<2 Q+2$.

It will be noted that the transformation applies in some cases where there are three equilibrium solutions of equation (1.2). In Figure 1 the appropriate values of $Q$ and $A$ lie in the gap between $A=A_{0}(Q)$ and $A=2 Q+2$ and each equilibrium solution of equation (1.2) maps into an equilibrium solution of equation (1.1), with the same value for the STF. The argument concerning the STF can easily be extended to the mapping of a $C 2$ solution but the restriction on $A$ means that $A<2 Q+2<A_{1}(Q)$ so we do not have to consider $C 2$ solutions in this case.

The case where $c=-1$ goes through in much the same way, although we have to distinguish the case $0<Q<1$ from the case $Q>1$. For $0<Q<1$, the appropriate transformation is

$$
y_{n}=\frac{4 x_{n}-2 A-4 Q}{\left(x_{n}-1\right) M_{1}}
$$

where

$$
M_{1}=\sqrt{ }\left\{(2-2 Q)^{2}-A^{2}\right\},
$$

and $A$ satisfies the restriction $0<A<2-2 Q$. With this transformation, $y_{n}$ satisfies equation (6.15) with

$$
M_{1} m=A+2+2 Q, \quad M_{1} k=4 A /(A+2-2 Q) .
$$


For the case $Q>1$, we can again use $M_{1}$ but the restriction on $A$ is now $0<A<2 Q-2$ and the sign on the right-hand sides of equations (6.20) and (6.22) has to be reversed. As in the case $c=1$, these transformations map equilibrium points into equilibrium points, with the same STF, although the restriction on $A$ limits their usefulness.

One property of equation (1.1) which can be used is the result, essentially due to Coppel [6], that the iteration process gives monotonic convergence to a limit when $0<2 k<m$. The limit must be a stable equilibrium point, with $S_{1}>0$ to ensure the monotonic behaviour. For $c=1$, equations (6.18) show that this condition is satisfied if

$$
0<\frac{8 A}{2+2 Q+A}<A+2-2 Q
$$

which is equivalent to

$$
4 Q^{2}<(A-2)^{2} \text {. }
$$

If we take $A>2$, equation (6.24) gives $A>2+2 Q$, which contradicts the restriction $0<A<2+2 Q$, so we must take $0<A<2$ in equation(6.24) and the requirement becomes $0<2 Q<2-A$ or $0<A<2-2 Q$. This implies that $0<Q<1$ and that we are dealing with points in the triangular region in Figure 1 defined by $A>0, Q>0$ and $0<A<2-2 Q$. The critical curve $A=A_{0}(Q)$ divides the triangle into two regions, with a single equilibrium solution for $0<A<A_{0}(Q)$ and three equilibrium solutions for $A_{0}(Q)<A<2-2 Q$. Where there is only one equilibrium solution it must be the positive solution and the monotonic convergence property shows that $0<S_{1}<1$ for this solution. From equation (2.5), this implies that $0<X_{3}<1$ for this equilibrium solution. (We know already that $S_{1}=0$ and $X_{3}=1$ when $A=2-2 Q$.) Where there are three solutions, with $X_{1}<X_{2}<0<X_{3}$, we know that the $X_{2}$ solution is always unstable so monotonic convergence implies $0<S_{1}<1$ for the other two solutions and hence $0<\left|X_{1}\right|<1$ and $0<X_{3}<1$.

We can extend this result a little further and state that $X_{3}>1$ except in the triangular region defined above. For a given value of $Q$ and $A$ large, equation (2.2) gives $X_{3} \sim \sqrt{A}$, so $X_{3}>1$ for $A$ large. We have $X_{3}=1$ only on the line segment $A=2-2 Q$ (with $0<Q<1$ ), so the change from $X_{3}<1$ to $X_{3}>1$ can only occur at points along this line. This implies that for points outside the triangular region $X_{3}>1$ and $-1<S_{1}<0$ for the corresponding value of $S_{1}$. (As $A \rightarrow \infty, S_{1} \rightarrow-1$ from above).

If we look at the transformations with $c=-1$ and see if the condition $0<2 k<m$ can be satisfied, equations (6.22) again give $4 Q^{2}<(A-2)^{2}$ and this leads to the same conclusions as before. For the case where $Q>1$, with $0<A<2 Q-2$, the equation for $m$ is $M_{1} m=-(A+2+2 Q)$ and hence $m<0$. Thus the condition $0<2 k<m$ cannot be satisfied. 
An unexpected consequence of this work on bilinear transformations is that in some cases equation (1.1) can be transformed into another equation of the same form. For example, if we take $A=2$ and $Q=3$ we have $0<A<2 Q+2$ so we can use equations (6.17) and (6.18). This gives

$$
y_{n}=\frac{2}{\sqrt{15}}\left(\frac{x_{n}-2}{x_{n}+1}\right), m=-\frac{1}{\sqrt{15}}, k=\frac{2}{5 \sqrt{15}} .
$$

On the other hand, $Q>1$ and $0<A<2 Q-2$, so we can use the second of the two cases for $c=-1$. This gives

$$
y_{n}=\frac{2}{\sqrt{3}}\left(\frac{4-x_{n}}{x_{n}-1}\right), m=-\frac{5}{\sqrt{3}}, k=\frac{2}{\sqrt{3}} .
$$

Thus two different versions of equation (1.1) map into the same version of equation (1.2) and it follows that they must map into each other.

This argument applies for any values of $Q$ and $A$ in the region $Q>1$, $0<A<2 Q-2$, that is, below the line $A=2 Q-2$ (for $Q>1$ ) in Figure 1. In the same way, we can use the transformation for $c=1$ and the first of the two cases for $c=-1$ in the triangular region $0<Q<1,0<A<2-2 Q$ in Figure 1. This includes some values of $Q$ and $A$ for which equation (1.2) has three equilibrium solutions.

If we go back to our example and use $w_{n}$ instead of $y_{n}$ in equation (6.26), to distinguish the two cases, we can eliminate $x_{n}$ and get

$$
\left(y_{n}+\frac{1}{\sqrt{15}}\right)\left(w_{n}+\frac{5}{\sqrt{3}}\right)=\frac{3}{\sqrt{5}} .
$$

This suggests that if we want to transform the equation

$$
y_{n+1}=2 k_{1} /\left\{1+\left(y_{n}-m_{1}\right)^{2}\right\}
$$

into a similar equation

$$
w_{n+1}=2 k_{2} /\left\{1+\left(w_{n}-m_{2}\right)^{2}\right\}
$$

we should try a relationship of the form

$$
\left(y_{n}-m_{1}\right)\left(w_{n}-m_{2}\right)=C,
$$

where $C$ is a non-zero constant. If we start from equation (6.28) and replace $y_{n}$ by $w_{n}$, from equation (6.30), then to obtain the equation for $w_{n}$ in the prescribed form we must have

$$
\begin{gathered}
m_{2}\left(m_{1}-2 k_{1}\right)=C, \\
m_{1}-2 k_{1}=m_{1} C^{2}, \\
m_{1} m_{2}-2 m_{1} k_{2}=C .
\end{gathered}
$$


From equation (6.32), $m_{1}=0$ is not admissible. Subtracting equation (6.33) from equation (6.31) gives

$$
m_{2} k_{1}=m_{1} k_{2}
$$

which implies that $m_{1}$ and $m_{2}$ have the same sign. For $m_{1}>0$, equation (6.32) shows that $m_{1}>2 k_{1}>0$ and it follows from equation (6.34) that $m_{2}>2 k_{2}>0$ in this case. If we put $m_{1}-2 k_{1}=m_{1} C^{2}$ in equation (6.31), we get

$$
C=1 /\left(m_{1} m_{2}\right)
$$

and equation (6.31) then gives

$$
m_{2}^{2}=\left\{m_{1}\left(m_{1}-2 k_{1}\right)\right\}^{-1} .
$$

If $m_{1}<0$, we must take $m_{2}<0$ in using equation (6.36) and the appropriate values of $k_{2}$ and $C$ follow from equations (6.34) and (6.35). Since there are no restrictions on $k_{1}$ when $m_{1}<0, k_{1}$ can be taken large enough to ensure that equation (6.28) has a stable $C 2$ solution and this $C 2$ solution maps into a $C 2$ solution of equation (6.29), with the same STF.

\section{Discussion of results}

It has been assumed throughout that $A$ and $Q$ are both positive and with this assumption appropriate boundary curves $A=A_{0}(Q), A=A_{1}(Q), \ldots A=A_{4}(Q)$ have been established for equation (1.2), as a guide to changes in the type of solution available. Once these. boundary curves are known it is fairly easy to construct one-parameter equations to illustrate different types of behaviour as the parameter increases. For example, if we take $A=10$ and let $Q$ vary there will be a range of values of $Q$ (roughly, $1<Q<3.5$ ) for which stable $C 2$ solutions occur but only equilibrium solutions would appear on the iteration diagram for other values of $Q$. There would be no solutions with period greater than 2 in this case. (Compare Figure 2.)

For $A=14$ and $Q$ as the parameter, solutions with period three exist for, roughly, $3<Q<5$ and this implies that solutions of all periods exist in this interval. For the $C 3$ solutions there will be two small intervals of stability, one close to $Q=3$ and the other close to $Q=5$, with a chaotic region in between. As $Q$ increases beyond $Q=5$ there would be a complete and fairly rapid reversal of the usual "period-doubling route to chaos". By the time $Q=6$ in this case we have $A=2 Q+2<A_{1}(Q)$ which means that we are back to equilibrium solutions and any further increase in $Q$ would not change this.

A more artificial example would be to join the point $(6,9)$ in the $(Q, A)$ plane to the point $(1,9)$ and to join $(1,9)$ to $(0.25,30)$, giving a piecewise linear relationship between $A$ and $Q$. In terms of Figure 2, this gives a path which starts 
below $A=A_{1}(Q)$, crosses it twice between $(6,9)$ and $(1,9)$ (so that it is below $A=A_{1}(Q)$ at $\left.(1,9)\right)$ and then crosses it twice more between $(1,9)$ and $(0.25,30)$. Where the path is below $A=A_{1}(Q)$ there are only equilibrium solutions; for the segments which are above $A=A_{1}(Q)$ there are stable $C 2$ solutions. If we use arc length along this path as the parameter (and choose appropriate values for $x_{0}$ ) there would be two bubbles on the iteration diagram, corresponding to the stable $C 2$ solutions. This illustrates a point made in Section 1 when discussing the paper by Oppo and Politi [10]. With a little more trouble in defining the relationship between $A$ and $Q$ it would be possible to have a curve which crosses $A=A_{1}(Q)$ several times but without crossing $A=A_{2}(Q)$. For example, we could use

$$
A(Q)=A_{1}(Q)+\frac{1}{2}\left\{A_{2}(Q)-A_{1}(Q)\right\} \sin 2 Q,
$$

with $2<Q<18$. If we take arc length along this curve as the parameter, equation (1.2) would give a series of bubbles on the iteration diagram. The main requirement is that the boundary curves should be known and the need for symmetry, which is sometimes specified, becomes less apparent.

It was partly to avoid using any symmetry properties that the conditions $A>0$ and $Q>0$ were maintained throughout Sections 2 to 6 . Solutions for $Q<0$ can easily be obtained from the solutions for $Q>0$, as pointed out by Bier and Bountis, but the behaviour for $A<0$ would have to be considered separately. The same approach could be used and a fair number of the equations would remain unchanged but appreciable changes in behaviour are to be expected in the solutions. To illustrate this we can take the case $Q=0$, which gives

$$
x_{n+1}=A x_{n} /\left(1+x_{n}^{2}\right) \text {. }
$$

For $A>0$ and $x_{0}>0$, it follows that $x_{n}>0$ for $n=1,2,3, \ldots$, whereas if $A<0$ and $x_{0}>0$ then $x_{n}$ changes sign at each iteration. If $x_{n}=X_{0}$ is a non-zero equilibrium solution for $A=A_{0}>0$, then $b_{1}=X_{0}, b_{2}=-X_{0}$ is a $C 2$ solution for $A=-A_{0}$. Indeed equation (7.2) cannot have a non-zero equilibrium solution for $A=-A_{0}$ although $C 2$ solutions exist, and are stable, for $A_{0}>1$. In contrast, for $A>0$ there are no $C 2$ solutions but non-zero equilibrium solutions exist, and are stable, for $A>1$.

\section{Acknowledgement}

This work was carried out as a Visiting Fellow in the Research School of Physical Sciences, Australian National University, and I am grateful to the Department of Theoretical Physics for the facilities it has provided. 


\section{References}

[1] M. Bier and T. C. Bountis, "Remerging Feigenbaum trees in dynamical systems", Phys. Lett. A. 104(1984), 239-244.

[2] A. Brown, "A nonlinear difference equation with two parameters", J. Austral. Math. Soc. Ser. $B 26$ (1985), 430-451.

[3] A. Brown, "A nonlinear difference equation with two parameters-II", J. Austral. Math. Soc. Ser. B 27 (1985), 145-166.

[4] J. Cascais, R. Dilao and A. Noronha da Costa, "Chaos and reverse bifurcation in a RCL circuit", Phys. Lett. A 93(1983), 213-216.

[5] P. Collet and J. P. Eckmann, Iterated maps on the interval as dynamical systems (Birkhäuser, Boston, 1980).

[6] W. A. Coppel, "The solution of cubic equations by iteration," Z. Angew. Math. Phys. 9a (1958), 380-383.

[7] T. Klinker, W. Meyer-Ilse and W. Lauterborn, "Period doubling and chaotic behaviour in a driven Toda oscillator," Phys. Lett. A 101(1984), 371-375.

[8] R.M. May, "Simple mathematical models with very complicated dynamics", Nature 261 (1976), 459-467.

[9] C. C. MacDuffee, Theory of equations (Wiley, New York, 1954), 77.

[10] G. L. Oppo and A. Politi, "Collisions of Feigenbaum cascades", Phys. Rev. A 30(1984), 435-441. 